\title{
Research on the Cultivation of Avocation Interest Relationship and Enhancement in the Sports Teaching and Sports Training
}

\author{
Yunna Zhao ${ }^{1}$ \\ ${ }^{1}$ Hebei Vocational\&Technical College of Building Materials, \\ Qinhuangdao,Hebei,066004 China
}

\begin{abstract}
In this paper, we conduct related research on the cultivation of avocation interest relationship and enhancement in the sports teaching and sports training. When studying initiative, even if encounter difficulties, will try hard to overcome which have the good mood. School physical education is the foundation of our socialist undertakings of physical culture and sports, sports teaching is an important part of school physical education. We firmly believe that through the reform according to the proposed methodology, the performance of the classes will be optimized.
\end{abstract}

Keywords: Avocation and Interest; Sports Teaching; Sports Training; Enhancement.

\section{Introduction}

In the sports teaching, paying attention to and develop the students' sports interest and ability is an effective guarantee to realize the goal of physical education curriculum and the value. Students for the upcoming whether to participate in desire and interest of learning, the direct impact on practice of the effect will be. Some students teaching content easily produce direct interest, and some content due to the mechanical repetition, dull, can let students psychologically boredom, avoid mood, etc. This requires the teacher according to the different situation of students correct guidance, cultivate and develop students' interests. This is complete the teaching task and improve the teaching effect is of great significance. We are interested in something happened, will concentrate and concentrate designed to do. In physical activity, are interested in is different with the result of a little interest, for the activities of the interest, can be persistent and focused attention. When studying initiative, even if encounter difficulties, will try hard to overcome which have the good mood. School physical education is the foundation of our socialist undertakings of physical culture and sports, sports teaching is an important part of school physical education. In the sports teaching to train students good study habits, to make the students know and attaches great importance to the development of sports for people on thought of huge role. This is not only promote the students to master basic sports knowledge and skills, but also promote students' physical and mental health development and the improvement of students' cultural knowledge level. New type of relationship between teachers and students should be a kind of based on democracy, equality, understanding and respect of a positive, cooperative relationship, cooperation should be a two-way, active, coordinated and harmonious, not tube and the tube. Teachers are not the leader but a mentor, not only is the age of the elderly, more should be a friend on equality of personality [1]. A new type of relationship between teachers and students is the foundation of good innovation environment.

With this foundation will everywhere inspire students' interest in creation and in a variety of innovative activity the aim is to improve the innovation ability of students. The characteristics of sports teaching and task analysis and the present sports teaching in our country's education career is the attention of the society, and it as a process of education, the characteristics of physical education teaching in a kind of nonverbal communication between 
teachers and students, teachers in the teaching process of explanation and demonstration to the corresponding teaching content, so as to guide students in the learning of corresponding to the corresponding sports skills and master a certain technology. In the process of education, not only to improve students' understanding of knowledge, and also required to realize the allround development of students, and sports as a basic motion to improve students' physical quality, as the development of the students to create a good physical quality [2]. Therefore, it is proved that the sports education to a great extent, is the cultivation of student's physical health to a certain, in enhancing students system at the same time, also has realized the student to the knowledge, technology and skills training, exercise science provides a good basis for the students. Starting from the characteristics of teaching, the implementation of the corresponding physical education task process to make a comprehensive consideration to the student's future and further development, completes the corresponding teaching purpose and should be carried out on the specific teaching content integration and implementation of the comprehensive, should also be the future of students interpersonal communication make the corresponding analysis, in order to promote and enhance personality development, communication and integration between the students and is in the process of sports teaching, to medium between teaching and learning to integrate and to constitute a good communication platform between teachers and students.

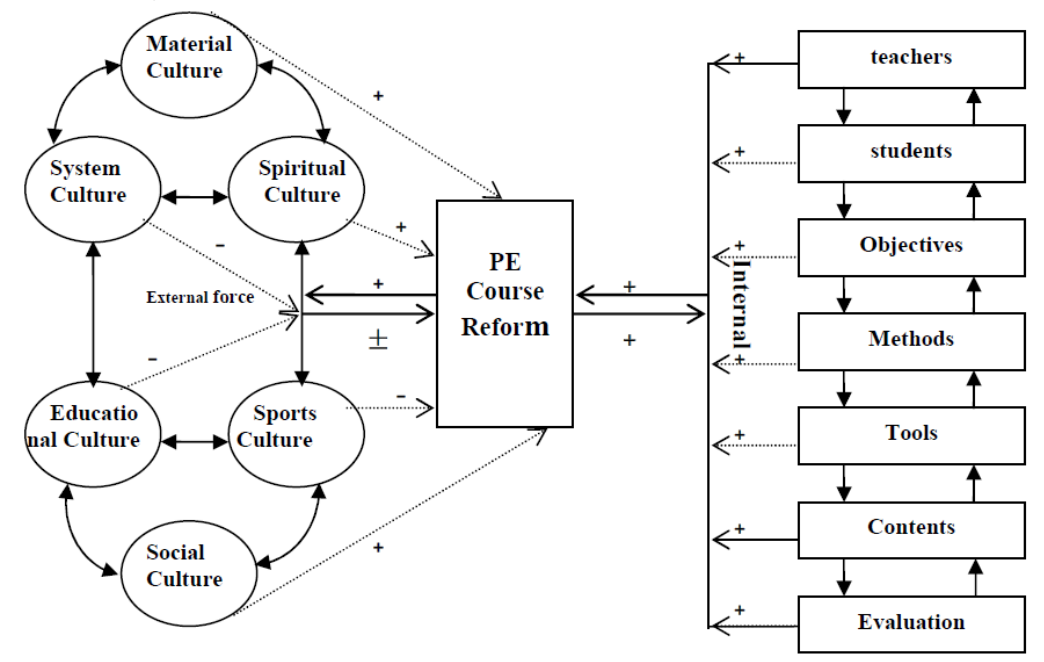

Figure 1. The Principles of the Enhancement in the Sports Teaching and Sports Training

In this paper, we conduct related research on the cultivation of avocation interest relationship and enhancement in the sports teaching and sports training. In the process of sports training, sports teaching corresponding principles to a great extent and there is a certain relationship between the corresponding training, principles of sports teaching in the process of the corresponding sports training is a reflection of a certain of this, it also suggests that exercise training is a certain scientific, main show is in the process of training the use of the corresponding training methods and means, on this basis, also means that the sports teaching the scientific principles for sports training provides a good quality assurance, therefore in the process of sports training, need to the corresponding principle of using teaching methods in order to achieve the effect of exercise training.

\section{Our Proposed Methodology}

The Sports Teaching and Sports Training. In colleges and universities sports teaching, physical education and sports coaching form the 
same and similar nature, but again there is essential difference between both tasks and purpose. Using competition to achieve specific purposes, and to fully embody the differences, which can maximally excavate potential, finally win in the competition. One of the specific process of the implementation of school education is embodied in physical education teaching, the use of specific teaching activities to guide students independent learning, which has been improved in terms of creativity and imagination and breakthrough, and in the lifelong physical exercise cultivate lifelong sports idea, good master specific skills, improve the level of physical quality and skills. Can take some of the reasonable and effective means of combination of infrastructure equipment and effective training methods to improve the quality of training, and improve the athletes' physical quality, explore its potential movement. In addition also can moderate increase the intensity of training in sports training, to improve performance on the basis of the original. People in sports teaching of physical exercise to study sports training sports competitions classify in sports, to them and the position of the relationship between the relatively few studies the boundary between them is fuzzy concept is not clear [3].

School sports teaching work to develop smoothly, competitive sports training must be constant attention, and school physical education and sports training are an organic whole, relying on each other. Out of the sports training school sports teaching is undoubtedly a backwater and lifeless, more the lack of the necessary passion and aesthetic feeling, is difficult to effectively arouse the enthusiasm of the students. At the same time, always emphasized on the basis of the physical education teaching in sports training, without sports teaching, sports training must also not good, become a madrassa reflected. Physical education and sports training should be synchronous development, lay the foundation for the development of competitive sports, sports teaching and the use of classroom teaching and extracurricular sports competition and sports training, and other methods to strengthen the students' sports consciousness and skill levels, make its of sports have a more profound and correct understanding, to cultivate a large number of high movement technical level of sports top and activists, to athletics sports reserve talented person in the future. In the process of sports teaching, combined with the corresponding training are needed to achieve the ultimate goal of teaching, in the process of sports training, however, need to the corresponding sports teaching content to ductility is applied, in order to achieve scientific sports training. It shows that both in the education process of penetration and connection, but also proves the existence between the two is inseparable relationship. In physical education and sports training, there are always some sports factors in teaching with the combination of education, and for sports training the ultimate goal is to achieve the relevant content.

The Cultivation of Avocation Interest Relationship and Enhancement. Interest will generate the corresponding motivation. Good teachers should be based on students' motivation to give guidance and help, help them develop short-term goals and long-term planning, using a variety of training means and methods, arouse their curiosity and desire to climb to the high level. The cultivation of interest is gradually and first gives a certain amount of stimulation, when he accepts the stimulation to the desired goal will generate incentives to achieve this goal. Motivation is the motive force of pushing it achieves the goal. Teachers in helping to achieve this goal, it will give certain stimulus again, when he has difficulty to produce negative emotions, stalled, teachers will be much help, encourage and guide them towards a positive direction to the enterprise. But interest in culture will vary from person to person, to pay attention to the method of stimulating and exciting. In the process of training, the teacher should pay attention to cultivate the feedback 
information of interest, to have keen powers of observation to understand the different age of the students' inner world. To different students use different methods to cultivate their interest in the specialty of the students.

Based on the essence of teaching and sports, sports teaching is under the physical education teachers' and students' participation, through the appropriate method, to guide the students to master the movement of sports and health knowledge, basic technology and skills, strengthen students' physique, train the ability of sports and the good thought personal character of an organization's education process and sports training is on the basis of the physical education teaching, the implementation of sport purpose, tasks, one of the main ways, is under the guidance of the coaches and athletes to actively participate in, to improve or maintain the performance of athletes and special organization of a kind of education process. Along with the development of modern education, people are also more and more attention to interest and emotional, students only by their interest in sports training, to affection is blended in among them, it will improve the quality of sports teaching, so the cultivation of emotion and interest in and transfer is a key factor in sports teaching. Teachers have a responsibility and obligation to arouse the enthusiasm of each student, inspire curiosity about things, resulting in a desire to ascertain, naturally in the gradual will generate strong interest. Starting from the characteristics of physical education teaching, based on the implementation of the physical education teaching task, you can see the whole process of sports teaching, must meet the following four basic factors. (1) As part of the sports science, although both embodies the essence of sports from different angles, but on the performance characteristics and content, is a significant difference. (2) Media, even though the sports teaching orderly systems in a certain time and space, make the information channel formed between synchronous coordinate activities between teachers and students. (3) Closely related to physical education teaching in sports training, broadly speaking, is to cultivate the athletes to create excellent grades, a complete and systematic process is based on physical activity with the purpose of the training process. (4) Physical education teaching principles, reflects the objective law of sports teaching, is the summary of experiences in sports teaching and generalization.

The Future and the Prospect. Reclassified to sports skill, on the basis of analyzing from the Angle of sports teaching motor skill learning rule, and according to the new classification of different types of sports skill in the process of learning the present a special law, law of motor skill learning process for the further research in the future to provide theoretical basis, and provide guidance for sports teaching workers choose teaching strategy. Choose the classification of motor skill as a focal point in the research of is due to a general study on the practical application of a wide range, more valuable than the study of the specific skills. Ready to exercise, depends on the people reflect some functional status and the objective external environment condition, should according to the teaching material content, environmental characteristics and strengthen training content and object. As time is short, all kinds of project teaching, training intensity big, must adopt corresponding countermeasures, should make physical activity fully open, keep the muscle ligament suddenly in a state of extreme stress, preparation of activity time will also increase.

Respect students' individual differences in sports teaching, for part of the constitution is poorer, athletic ability weaker students to discriminate, to ensure that every college students in physical education teaching. In addition, should be aimed at college students' individual differences in scientific, rational analysis, combined with the layered teaching mode, for each of the college students to make the most suitable sport plan. Some college students lack of exercise, for example, passion, 
and all energy on academic study, sports participation enthusiasm is very low however, this part of the college students' thinking ability is stronger, good at thinking, study is one of their advantages, this part in PE teaching in colleges and universities can be combined with the characteristics of college students, to develop some interesting sports teaching. Thus, on the one hand, to enhance the sports teaching the lively, vivid, to improve the students' interests in participation, on the other hand, it is helpful for students' thinking ability of exercise, improve the sports motivation and sports ability.

\section{Conclusion}

In this paper, we conduct related research on the cultivation of avocation interest relationship and enhancement in the sports teaching and sports training. Needed for successful teaching is not mandatory, but arouse the students' interest in learning. Efforts to stimulate and cultivate the students' interest in learning, to make the students enjoy the fun of learning which is one of the tasks of sports teaching under the new curriculum idea. Teachers are not the leader but a mentor, not only is the age of the elderly, more should be a friend on equality of personality. Students have emotional needs, starting on the first day of PE class and they need to get respect from teachers, love, warmth, friendship, and taught. When this kind of emotional need is met, they will with greater passion for gym class. Through the proposed approaches, the performance of the classes will be enhanced.

\section{References}

[1] Hou K. Intervention study of sports injuries on physical education teaching and training for professional football sports[J]. Shandong Sports Science \& Technology, 2009.

[2] Chen D, Deng H. Application of Inquiry Teaching in the Tennis Teaching of Sports Training and Technical College[J]. Guide of Science \& Education, 2011.

[3] Zhang S Y, Lin Y. Study on Professional Track and Field Sports Training Special Theory and Practice Teaching Evaluation Reform[J]. Sports Science Research, 2012. 